\title{
Nutritional support via percutaneous endoscopic gastrostomy
}

\author{
BY FIONNUALA M. DOYLE* AND NICHOLAS P. KENNEDY \\ Unit of Nutrition and Dietetic Studies, Department of Clinical Medicine, Trinity Centre for Health \\ Sciences, St James's Hospital, Dublin 8, Republic of Ireland
}

Nutritional support is often an integral part of patient management. There are three principal access routes used for nutrient delivery: oral, enteral (tube feeding) and parenteral.

Tube feeding is indicated for those patients who, in the presence of a functional gastrointestinal tract, cannot, should not or will not consume adequate nutrition orally. A number of different routes may be used in administering enteral feeds, which include nasogastric (NG), nasojejunal and enterostomy (e.g. percutaneous endoscopic gastrostomy (PEG)) methods. PEG feeding is the main topic of the present review.

The concept of nutritional support via gastrostomy was first proposed by a Norwegian surgeon, Egeberg, in 1837 and first performed on the animal model by Sedillot in 1839 . In 1876, Verneuil successfully performed a surgical gastrostomy in man. It was the advent of the flexible endoscope a century later that enabled the percutaneous endoscopic technique to be developed. This was first described and performed by Guaderer and Ponsky late in 1979 (Lo \& Dornbrand, 1989).

\section{NUTRITIONAL SUPPORT}

When it is judged that a patient requires nutritional support, following a full nutritional assessment (Thomas, 1988), a decision must be made concerning how the required nutrients should be delivered. Where possible, use of the oral route is preferable (Jayson-Payne, 1992). In this case, energy and nutrient intake can be manipulated using energy- and nutrient-dense foods, frequently in association with nourishing drinks (usually commercially prepared) with or without supplements. Food and fluid texture may need to be altered, particularly in the dysphagic patient, to reduce the dangers of aspiration by enhancing safer swallowing.

If oral intake is inadequate or contraindicated and the gastrointestinal tract is functional, then enteral tube feeding is preferable to parenteral nutritional support, due to the higher complications and costs associated with the latter (Armstrong et al. 1990). The specific route chosen to deliver enteral feeds will depend on the following: (1) the presence of a self-limiting illness which may only require short-term feeding, (2) the presence of chronic pathology which may necessitate long-term or indefinite nutritional support (Jayson-Payne, 1992).

Until recently, NG feeding has been implemented for both short-term and long-term tube feeding. However, a number of problems are associated with the use of NG tubes over the longer term (for example, 4 weeks or more). These include nasal and oral discomfort (particularly with Ryles tubes), tube blockage and unplanned extubation by patient or staff. Should recurrent intubation be necessary, this increases the risks of

* Present address: Rowa Pharmaceuticals, Bantry, County Cork, Republic of Ireland. 
Table 1. Specific indications for percutaneous endoscopic gastrostomy (PEG) feeding

1. Neurological disorders or trauma Stroke

Head injury

Hemiplegia

Degenerative diseases of the CNS

Other neurological conditions

2. Oropharyngeal disorders Cancers of the head and neck

3. Supplemental feeding Cystic fibrosis AIDS
4. Intensive care

5. Tracheo-oesophageal fistulas

6. Rare indications

Short bowel syndrome

Gastric decompression

Recurrent aspiration (on NG feeding)

Inflammatory myopathy

Connective tissue disease

Macroglossia secondary to amyloid

Severe burns

CNS, central nervous system; AIDS, acquired immune deficiency syndrome; NG, nasogastric.

oesophageal perforation and malpositioning complications (Jackson et al. 1990). In addition, many patients or their families object to the physical presence of a nasoenteric tube.

When the patient is expected to need enteral nutritional support for a long period, PEG has clear advantages over the nasoenteric routes (Larson et al. 1987; Johnston et al. 1993). PEG tubes are rarely removed inadvertently, therefore the patient is more likely to receive the prescribed quantity of nutrients and, thus, achieve the desired objectives of weight gain and improved nutritional status (Forgacs et al. 1992; Park et al. 1992). Once the stoma has healed, PEG tubes cause little or no physical discomfort. In addition, a PEG tube can remain in situ for up to 3 years, a more desirable alternative than recurrent nasogastric intubation. A decreased risk of aspiration has been reported (Ciocon et al. 1990), although this is not universally agreed (Jarnagin et al. 1992). As the PEG tube is concealed by clothing when not in use, it is less obtrusive than the nasoenteric alternatives.

As a rule, PEG is preferred to surgical gastrostomy because it is a minimally invasive surgical procedure, is more cost effective and has fewer associated complications (Moran et al. 1990; Sali et al. 1993).

\section{INDICATIONS FOR PERCUTANEOUS ENDOSCOPIC GASTROSTOMY FEEDING}

PEG feeding can be used in a wide variety of disease states (see Table 1), but these can be categorized broadly into: (1) patients having reversible disease with potential for recovery, (2) patients suffering from incurable diseases with potentially extended survival, (3) patients who are terminally ill or seriously debilitated (Wolfson et al. 1990a).

PEG feeding is most frequently used for patients who cannot eat enough to meet their needs, primarily those with neurological disorders and associated dysphagia (Allison et al. 1992), such as stroke (Roukema et al. 1990; Sali et al. 1993), head injury or trauma (Larson et al. 1987), degenerative diseases of the central nervous system (Raha et al. 1991) and cancer of the head and neck (Fietkau et al. 1989). PEG is also effective for supplemental feeding in conditions such as cystic fibrosis (Steinkamp et al. 1990) and acquired immune deficiency syndrome (AIDS; Kotler et al. 1991). 
Table 2. Specific contraindications to percutaneous endoscopic gastrostomy (PEG) feeding

1. Contraindications to endoscopy

Severe tumours of the head and neck

2. Contraindications to gastrostomy

Massive obesity

Massive ascites

Peritoneal dialysis

Peritonitis

Acute pancreatitis

Portal hypertension

Coagulation disorders

3. Contraindications to gastric instillation of feeds

Severe gastro-oesophageal reflux

Gastric outlet obstruction

\section{CONTRAINDICATIONS TO PERCUTANEOUS ENDOSCOPIC GASTROSTOMY FEEDING}

PEG feeding is unsuitable for cases where: (1) oral intake is sufficient or where nutritional support is only required for short periods (for example less than 4 weeks), (2) enteral feeding is contraindicated, (3) the patient or the next-of-kin (when the patient is unable to communicate) objects (Lo \& Dornbrand, 1989), (4) the patient is terminally ill (Wolfson et al. 1990b), (5) endoscopy is impractical or contraindicated (O'Dwyer et al. 1990).

\section{PLANNING THE PERCUTANEOUS ENDOSCOPIC GASTROSTOMY NUTRITIONAL SUPPORT REGIMEN}

The process of planning the enteral nutritional support regimen is the same, regardless of the access route chosen. Individual energy, protein, nutrient and fluid requirements are assessed using standard formulas (Thomas, 1988). Based on the calculated requirements, the appropriate feeding formula is selected. This is generally a $4.2 \mathrm{~kJ} / \mathrm{ml}$ polymeric feed, frequently administered in volumes of $2 \cdot 0-2 \cdot 5 \mathrm{l} / \mathrm{d}$, with a fibre content varying from $0-15$ $\mathrm{g} / \mathrm{l}$. Commercial formulas are available to cater for a wide range of energy, protein and electrolyte requirements.

Partially or fully predigested protein feeds, may be used if the access route is to the lower duodenum or jejunum, or if there is a malabsorptive syndrome. The method by which the feeding formula is delivered varies from one hospital to another. Many choose continuous feeding with pump assistance (although a pump is not essential). Feeding is commenced at reduced rates and increased over $2-3 \mathrm{~d}$ to allow delivery of the daily target volume (the rate of increase varies between patients according to individual tolerance). Peripheral fluids are simultaneously administered at reduced rates and discontinued when the target enteral feed volume is achieved. Alternatively, some choose to vary the concentration of feed rather than the rate of its delivery, beginning the regimen with 
diluted feeds and increasing their concentration over several days. This approach has financial and practical benefits, as it reduces or eliminates the necessity for concurrent administration of peripheral fluids. Although referred to as continuous feeding, a common practice is to administer the enteral nutrition solution in several feeding periods. Each feed may last $6-8 \mathrm{~h}$, such that the duration of feeding may last between 16 and $24 \mathrm{~h} / \mathrm{d}$. This type of feeding protocol allows tight control of the amount and rate of feed delivered, whilst requiring less nursing time than bolus feeding. It is also particularly suitable for patients with a tendency to gastro-oesophageal reflux.

Bolus feeding is often preferred by patients and staff because it mimics the normal eating pattern more closely and it allows independence from pumps. Many alert and mobile patients feel that it is user-friendly and suits their lifestyle. However, where unconscious or immobile patients are concerned, bolus feeding is labour-intensive for the carers. It is important that the feeding method chosen (continuous or bolus) should suit the hospital or home routine and that the patient's preference be considered.

Feeding is often commenced $12 \mathrm{~h}$ after insertion of the PEG tube, although some centres delay this for up to $24 \mathrm{~h}$, preferring to flush the tube with either saline or dextrose solution at regular intervals (for example, at intervals of $6 \mathrm{~h}$ ).

\section{MONITORING}

Careful clinical, anthropometric, haematological and biochemical monitoring of the patient receiving enteral nutritional support is important in evaluating the efficacy of a feeding regimen and the patient response to therapy. Clinical assessment should include measurement of vital signs and checking for signs of gastrointestinal intolerance (nausea, vomiting, diarrhoea, bloating and abdominal distension). Until the patient's condition has stabilized, fluid balance and bowel activity should be assessed daily. The position of the tip of the PEG tube should be checked occasionally by aspirating lumen fluid and checking for gastric acid. The patient's level of satisfaction with the nutritional therapy should also be monitored.

Anthropometric variables, including body weight, should be measured periodically. Where practicable, this should be carried out at weekly intervals initially, then monthly for patients in hospital and every 3-6 months for those in the community.

Haematological and biochemical monitoring, which should be performed at similar intervals, usually consists of a full blood count and measurement of serum electrolytes, glucose, urea, albumin and transferrin. It may be necessary to measure trace elements, folate and vitamin $\mathrm{B}_{12}$ monthly in patients who are at higher risk of micronutrient deficiency (such as the elderly or those with chronic malabsorptive conditions).

\section{COMPLICATIONS AND PREVENTION OF PROBLEMS DURING PERCUTANEOUS ENDOSCOPIC GASTROSTOMY FEEDING}

Major complications (see Table 3) with the use of PEG feeding are uncommon; incidence rates of 1-3\% over variable periods of time (Gay et al. 1992) and $30 \mathrm{~d}$ mortality rates in the region of $1 \%$ (Hollands et al. 1989) have been reported. However, the rate of minor complications (such as reflux aspiration, gastrostomy wound infection, tube blockage or rupture) is more significant (Seyrig et al. 1990), with some authors reporting 
Table 3. Complications of percutaneous endoscopic gastrostomy (PEG) feeding

1. Major
Aspiration pneumonia
Gastric perforation
Gastric bleeding
Haematoma

1. Major

Aspiration pneumonia

Gastric bleeding

Haematoma

\author{
2. Minor \\ Pyrexia \\ Wound infection \\ Abscess of the abdominal \\ wall \\ Stomal leaks \\ Tube migration \\ Auto-extubation \\ Aspiration of feeds \\ Anorexia
}

3. Late

Catheter displacement or rupture

Local peritonitis

Respiratory problems

Tube impaction

rates as high as $13 \%$ (Hull et al. 1993). Complication rates are likely to be higher if PEG feeding is used in unsuitable patients and, therefore, can be expected to decline as PEG usage becomes more established.

Education of both carers and patients is essential for the successful application of PEG feeding and to reduce unnecessary complications. Carers should be educated about all aspects of PEG before they assume the care of patients on PEG feeding. Patients should be educated about PEG tubes, their care and the feeding regimen before tube insertion. At present, it is unfortunate that appropriate and well-timed education is not always provided.

\section{GASTROSTOMY-RELATED PROBLEMS}

The short-term incidence of wound infection is frequently as high as 6\% (Hull et al. 1993). Prophylactic administration of a single intravenous dose of a broad-spectrum antibiotic before tube insertion can help to prevent wound infection. Following the insertion of a PEG tube, the stoma is covered with an antiseptic dressing until it heals (usually no more than 10-12 d). Once healing is complete, the stoma should not be considered a wound and need not necessarily be dressed, although some leakage of gastric fluid around the tube may be experienced. Consequently, the site should be checked daily for rash, irritation and tenderness. The peristomal region should be washed daily with soap and water and dried thoroughly. The tube should be routinely partially rotated in situ to assess whether or not there is deep tenderness and to minimize the likelihood of epithelialization of the internal flange (Lipscomb et al. 1994).

Blockage of the tube has been reported to occur in 2-18\% of patients on PEG feeding. This can occur as an early or a late complication (Hull et al. 1993). Blockages are more likely to occur if staff have insufficient training or experience in using PEG tubes, or if the patient has not been adequately educated about care of their tube. Blocked tubes are more likely to rupture, to need replacement and to be a source of infection (Gottlieb et al. 1993). Tube blockage can be prevented by applying simple measures such as limiting the use of the tube to fluids (feeding formulas, water, liquid medications) and flushing the tube before and after each feed. Should the tube become blocked, it may be cleared by gently administering approximately $50 \mathrm{ml}$ water, a carbonated beverage (e.g. 7-Up), diluted vinegar, diluted lemon juice, or bicarbonate of soda using a $20 \mathrm{ml}$ or $50 \mathrm{ml}$ syringe. 
Tubes with significant extra-corporeal length should be taped securely to the abdominal skin surface (to avoid accidental dislodgement). When the tube is not in use it should be closed (using the clamp or stopper provided in the PEG kit).

\section{GASTROINTESTINAL PROBLEMS}

Many patients suffer from a dry mouth (xerostomia) and poor oral hygiene during PEG feeding because their oral intake is minimal or non-existent. The xerostomia may be worsened by concomitant use of drugs which decrease salivation (e.g. sedatives) and by the hospital environment, which tends to be warm and dry. This oral dryness and accompanying halitosis can cause considerable discomfort to the patient. There are now a number of commercially-available artificial salivas (presented as sprays) which can be very helpful in the treatment of these symptoms. The artificial salivas moisten the mouth but do not stimulate salivation and, therefore, are suitable for dysphagic patients (in whom excessive saliva may be inhaled). Inhalation of saliva or feeds into the lungs may result in aspiration pneumonitis or pneumonia. Gastro-oesophageal reflux and pulmonary aspiration of feeds is one of the more frequent complications of NG or PEG feeding. Patients who have a known history of aspiration or gastro-oesophageal reflux, or who have an increased risk of oesophageal reflux (elderly, immobile or bedridden patients), or who are noted to have oesophagitis during PEG placement, are more likely to suffer from aspiration (Patel \& Thomas, 1990). Preventative measures, which may reduce the incidence of aspiration but will not eliminate it, include elevation of the bed-head to $30^{\circ}$ from the horizontal and positioning the patient in an upright position during feeding (particularly if bolus feeding). If gastric emptying is delayed, the rate of continuous feeding or the timing of bolus feeds may need to be adjusted. Delaying feeding (by $1-3 \mathrm{~h}$ ) has been recommended if the gastric residue is found to be more than $100 \mathrm{ml}$ (Metheny et al. 1993). Alternatively, a prokinetic motility agent may be used. If a patient is prone to aspiration, it may be preferable to use a longer tube through the percutaneous gastrostomy, allowing placement of the tube tip into the jejunum (DiSario et al. 1990). In some cases, it may be necessary to consider using a concurrent tracheostomy (e.g. in unconscious patients; Wolfson et al. 1990b).

A significant number of enterally-fed patients experience diarrhoea. The enteral feed is often not the cause. If the diarrhoea is associated with the initiation of a feeding regimen, it is more likely to be associated with enteral feeding (feeds are too concentrated or delivery rate is too fast, or a combination of both). In this case, the progression of the feeding regimen should be revised and the concentration or rate of delivery decreased. If these measures are insufficient to eliminate the symptom and if there is no other cause found (e.g. infection or change in underlying disease status), then intolerance to the feed formulation should be considered.

If a patient is established on feeding, the feeding formula has not passed its expiry date, and the flow-rate is not excessive, then the feeding formula is unlikely to cause diarrhoea. It is important to determine whether or not antibiotic treatment has recently been commenced, as this is a common cause of diarrhoea. If not, stools should be examined for evidence of an infective cause. If diarrhoea of unknown origin persists, a period of 'bowel rest', using partially or fully predigested protein feeds, may solve the problem. Following this, feeding with polymeric formulas may be re-introduced gradually. 
Constipation frequently occurs with all forms of enteral feeding, particularly PEG feeding, because a large number of the patients are on long-term feeding, are immobile, have had strokes and are consequently more likely to have reduced peristalsis. To compound this problem, such patients often have insufficient fluid and dietary fibre intakes. Constipation can be eliminated or diminished, avoiding the discomfort and humiliation of enemas and decreasing expenditure on laxatives. Sometimes, increasing fluid intake to an appropriate level will suffice. Fibre-containing feeds may be administered to provide up to $30 \mathrm{~g}$ fibre in a 2 litre feed. The use of the natural laxative, prune juice, if administered as a $30-50 \mathrm{ml}$ dose in the last feed at night, is very effective. Some patients with long-term constipation may be given prophylactic laxatives.

\section{HOME PERCUTANEOUS ENDOSCOPIC GASTROSTOMY}

A major advantage of PEG feeding is that its use makes it possible to discharge an increasing number of patients into the community or into nursing homes who, formerly, would have remained dependent on hospital in-patient care. It is crucial that such a transition is well organized, that only suitable patients are selected and, importantly, that the patient is in agreement (Ponsky et al. 1989).

Sufficient advance warning is advisable so that community systems can be established in good time. The patient (where possible) and family or carer should be educated about all aspects of PEG feeding and care. They should receive written (and pictorial if necessary) information for reference at home, which should include contact names and telephone numbers for emergency back-up.

The feeding regimen should be suited to the individual's lifestyle and requirements. Mobile young patients of stable weight and condition who have been established on PEG feeding may prefer the PEG feeding button (rather than tube) as it is unobtrusive, thereby enhancing the possibility of leading a more normal lifestyle. Requirements for a pump or other feeding equipment should be determined before discharge so that the patient and the carer(s) can be trained appropriately before the patient goes home. With the consent of the patient, the public health nurse should be alerted and home visits organized. The general practitioner (GP) should be informed of the patient's medical condition and all aspects of PEG and the feeding regimen (preferably in writing), because the GP is often the first person the patient goes to with a problem. The local pharmacy should be contacted to ensure that the necessary feeds and equipment are available.

Financial matters should be considered in advance of discharge. When the patient has a medical card, feeding materials and equipment will be provided free of charge if the items are approved by the General Medical Services (GMS) Scheme in the Republic of Ireland or the Advisory Committee on Borderline Substances (ACBS) in the UK. If the patient does not have a medical card, often refunds may be claimed from the local Health Board (e.g. under the Assistance Towards the Cost of Prescribed Medicine Scheme in the Republic of Ireland). Most patients who need home PEG will qualify for this type of benefit. For patients who are not eligible for such assistance, benefits may be claimed under other schemes, or tax relief may be available on health expenses which have not been reimbursed.

Follow-up care should be organized (if possible) before the patient is discharged from hospital, rather than arranging a review only when problems arise. A routine protocol 
for follow-up may prevent some of the avoidable problems and consequent unnecessary distress.

\section{DISCONTINUATION OF PERCUTANEOUS ENDOSCOPIC GASTROSTOMY FEEDING}

PEG feeding should be discontinued when oral intake is sufficient, if the enteral route becomes contraindicated, if a patient is terminally ill (when death is imminent), or at the patient's request, in some cases. PEG feeding may be temporarily stopped if the PEG tube is irreversibly damaged, whilst awaiting tube replacement. If a patient is terminally ill, and unfit for endoscopy, or if there is a temporary improvement in oral intake, the tube may remain in situ and be cared for in the usual manner (Cullado et al. 1990), but will not be used. In certain circumstances, moral or ethical issues may make the decision to continue or to terminate feeding a difficult one. When PEG feeding is no longer required, the tube is removed, usually by an endoscopic method (Wilson et al. 1990; Dilorenzo et al. 1992).

\section{CONCLUSION}

PEG is a reliable, safe and cost-effective method for long-term enteral feeding (Mohandas et al. 1992). The insertion procedure is minimally invasive and a PEG tube, if properly cared for, can remain in situ for up to 3 years (Dilorenzo et al. 1992). This method of feeding allows for an increasing number of hospital patients to be discharged into the community.

However, it is important that patients are carefully selected and that they give informed consent (Clarkson et al. 1990). If long-term nutritional support via PEG is to be successful, it is essential that patients and their carers are appropriately educated about PEG feeding and trained in the use of the tube and the necessary equipment. In view of the likely increase in numbers of patients being fed by PEG, the development of general protocols for procedures, feeding regimens and follow-up should be encouraged, for use both in hospitals and in the community. This would diminish confusion for patients and for staff as they move between hospitals.

In considering the use of PEG feeding in patient management, the patient's perspective must also be given credence, a point eloquently made by Lo \& Dornbrand (1989): 'It has been asserted that medicine should benefit the patient according to the patient's definition of benefit'.

\section{REFERENCES}

Allison, M. C., Morris, A. J., Park, R. H. R. \& Mills, P. R. (1992). Percutaneous endoscopic gastrostomy tube feeding may improve outcome of late rehabilitation following stroke. Journal of the Royal Society of Medicine 85, 147-149.

Armstrong, P. J., Hand, M. S. \& Frederick, G. S. (1990). Enteral nutrition by tube. Veterinary Clinics of North America Small Animal Practice 20, 237-275.

Ciocon, J. (1990). Indications for tube feeding in elderly patients. Dysphagia 5, 1-5.

Clarkson, W. K., Smith, O. J. \& Waldon, J. M. (1990). Percutaneous endoscopic gastrostomy and early mortality. Southern Medical Journal 83, 1433-1436.

Cullado, M. J., Slezak, F. A. \& Porter, J. A. (1990). Repeat percutaneous endoscopic gastrostomy: An outpatient procedure. Surgical Endoscopy 4, 173-174. 
Dilorenzo, J., Dalton, B. \& Miskovitz, P. (1992). Percutaneous endoscopic gastrostomy: What are the benefits, what are the risks? Postgraduate Medicine 91, 277-281.

DiSario, J. A., Foutch, P. G. \& Sanowski, R. A. (1990). Poor results with percutaneous endoscopic jejunostomy. Gastrointestinal Endoscopy 36, 257-260.

Fietkau, R., Thiel, H. J., Iro, H., Richter, B., Senft, M., Rossler, C., Kolb, S. \& Sauer, R. (1989). Comparison between oral nutrition and enteral nutrition using a percutaneous endoscopically-guided gastrostomy (PEG) in patients undergoing radiotherapy for head and neck tumors. Stralenther Onkologie 165, 844-851.

Forgacs, I., Macpherson, A. \& Tibbs, C. (1992). Percutaneous endoscopic gastrostomy. British Medical Journal 304, 1395-1396.

Gay, F., el Nawar, A. \& Van Gossum, A. (1992). Percutaneous endoscopic gastrostomy. Acta Gastroenterologica Belgica 55, 285-294.

Gottlieb, K., Leya, J., Kruss, D. M., Mobarhan, S. \& Iber, F. L. (1993). Intraluminal fungal colonization of gastrostomy tubes. Gastrointestinal Endoscopy 39, 413-415.

Hollands, M. J., Fletcher, J. P. \& Young, J. (1989). Percutaneous feeding gastrostomy. Medical Journal of Australia 151, 328-331.

Hull, M. A., Rawlings, J., Murray, F. E., Field, J., McIntyre, A. S., Mahida, Y. R., Hawkey, C. J. \& Allison, S. P. (1993). Audit of outcome of long-term enteral nutrition by percutaneous endoscopic gastrostomy. Lancet 341, 869-872.

Jackson, R. H., Payne, D. K. \& Bacon, B. R. (1990). Esophageal perforation due to nasogastric intubation. American Journal of Gastroenterology 85, 439-442.

Jarnagin, W. R., Duh, Q. Y., Mulvihill, S. J., Ridge, J. A., Schrock, T. R. \& Way, W. L. (1992). The efficacy and limitations of percutaneous endoscopic gastrostomy. Archives of Surgery 127, 261-264.

Jayson-Payne, J. (1992). Current concepts in clinical nutrition: assessing patients. Nutrition 8, 223-231.

Johnston, D. A., Anderson, J. T. \& Pennington, C. R. (1993). Percutaneous endoscopic gastrostomy (PEG): a major advance in enteral feeding. Scottish Medical Journal 38, 68-69.

Kotler, D. P., Tierney, A. R., Ferraro, R., Cuff, P., Wang, J., Pierson, R. N. Jr. \& Heymsfield, S. B. (1991). Enteral alimentation and repletion of body cell mass in malnourished patients with acquired immunodeficiency syndrome. American Journal of Clinical Nutrition 53, 149-154.

Larson, D. E., Burton, D. D., Schroeder, K. W. \& Di Magno, E. P. (1987). Percutaneous endoscopic gastrostomy: Indications, success, complications and mortality in 314 consecutive patients. Journal of Gastroenterology 93, 48-52.

Lipscomb, G. R., Brown, C. M., Wardle, T. \& Rees, W. D. (1994). Blocked gastrostomy tubes. Lancet 343, 801-802.

Lo, B. \& Dornbrand, L. (1989). Understanding the benefits and burdens of tube feeding. Archives of Internal Medicine 149, 1925-1926.

Metheny, N. (1993). Minimizing respiratory complications of nasoenteric tube feeding: state of the science. Heart Lung 22, 213-223.

Mohandas, K. M., Dave, U. R., Santhi Swaroop, V., Desai, D. L., Dhir, V., Pradhan, S. A., Bathena, H. M. \& Kavarana, N. M. (1992). Percutaneous endoscopic gastrostomy for long term enteral nutrition. National Medical Journal of India 5, 52-55.

Moran, B. J., Taylor, M. B. \& Johnson, C. D. (1990). Percutaneous endoscopic gastrostomy. British Journal of Surgery 77, 858-862.

O'Dwyer, T. P., Gullane, P. J., Awerbuch, D. \& Ho, C. S. (1990). Percutaneous feeding gastrostomy in patients with head and neck tumours: a 5 year review. Laryngoscope 100, 29-32.

Park, R. H. R., Allison, M. C., Lang, J., Spence, E., Morris, A. J., Danesh, B. J. Z., Russell, R. I. \& Mills, P. R. (1992). Randomised comparison of percutaneous endoscopic gastrostomy and nasogastric tube feeding in patients with persisting neurological dysphagia. British Medical Journal 304, 1406-1409.

Patel, P. H. \& Thomas, E. (1990). Risk factors for pneumonia after percutaneous endoscopic gastrostomy. Journal of Clinical Gastroenterology 12, 389-392.

Ponsky, J. L. \& Gauderer, M. W. L. (1989). Percutaneous endoscopic gastrostomy: indications, limitations, techniques and results. World Journal of Surgery 13, 165-170.

Raha, S., Finucane, P. \& Duncan, D. (1991). Percutaneous endoscopic gastrostomy. British Journal of Hospital Medicine 46, 53-55.

Roukema, J. A., Van der Werken, C. \& Juhman, J. R. (1990). Percutaneous endoscopic gastrostomy as a standard procedure in head and neck surgery. Archives of Otolaryngology - Head and Neck Surgery 116, $730-731$. 
Sali, A., Wong, P. T., Read, A., McQuillan, T. \& Conboy, D. (1993). Percutaneous endoscopic gastrostomy: the Heidelberg Repatriation Hospital experience. Australian and New Zealand Journal of Surgery 63, 545-550.

Seyrig, J. A., Gardin, J., Costa, B., Bussane, M., Berger, M., Bow, B., Hirsch, J. F., Tabouret, M., Mandelbaum, B. \& Bertrand, H. J. (1990). Percutaneous endoscopic gastrostomy: 174 cases. Presse Medicale 19, 1035-1039.

Steinkamp, G., Rodeck, B., Seidenberg, J., Ruhl, I. \& van der Hardt, H. (1990). Stabilization of lung function in cystic fibrosis during long-term feeding via percutaneous endoscopic gastrostomy. Pneumologie 44, 1151-1153.

Thomas, B. [editor] (1988). The assessment of nutritional status in clinical situations. In Manual of Dietetic Practice, pp. 59-65. Oxford: Blackwell Scientific Publications.

Wilson, W. C. M., Zenone, E. A. \& Spector, H. (1990). Small intestinal perforation following placement of a percutaneous endoscopic gastrostomy tube. Gastrointestinal Endoscopy 36, 62-63.

Wolfson, H. C., Kozarek, R. A., Ball, T. J., Patterson, D. J. \& Botoman, V. A. (1990a). Tube dysfunction following percutaneous endoscopic gastrostomy and jejunostomy. Gastrointestinal Endoscopy 36, 261-263.

Wolfson, H. C., Kozarek, R. A., Ball, T. J., Patterson, D. J., Botoman, V. A. \& Ryan, J. A. (1990b). Long term survival in patients undergoing percutaneous endoscopic gastrostomy and jejunostomy. American Journal of Gastroenterology 85, 1120-1122. 